\title{
Canine Liver Transplantation Model and the Intermediate Filaments of the Cytoskeleton of the Hepatocytes
}

\author{
Consolato Sergi, ${ }^{1,2}$ Reem Abdualmjid, ${ }^{1}$ and Yasser Abuetabh ${ }^{1}$ \\ ${ }^{1}$ Department of Laboratory Medicine and Pathology, University of Alberta, Edmonton, AB, Canada T6G $2 B 7$ \\ ${ }^{2}$ Department of Laboratory Medicine and Pathology, Walter Mackenzie Health Sciences Centre, 5B4.09, 8440-112 Street, Edmonton, \\ $A B$, Canada T6G 2B7
}

Correspondence should be addressed to Consolato Sergi, biotechlab@gmail.com

Received 10 December 2011; Accepted 27 January 2012

Academic Editor: Andrea Vecchione

Copyright ( 92012 Consolato Sergi et al. This is an open access article distributed under the Creative Commons Attribution License, which permits unrestricted use, distribution, and reproduction in any medium, provided the original work is properly cited.

Liver transplantation has been a successful therapy for liver failure. However, a significant number of recipients suffer from graft dysfunction. Considerably, ischemia and reperfusion (I/R) injury is the most important factor leading to organ dysfunction, although the pathogenesis has not been fully described. I/R injury have several established features that are accompanied by and/or linked to bile duct loss or ductopenia, cholestasis, and biliary ductular proliferations in the posttransplant liver biopsy. However, biliary marker levels increase usually only 5-7 days after transplantation. Intermediate filaments are one of the three cytoskeletal proteins that have a major role in liver protection and maintaining both cellular structure and integrity of eukaryotic cells. We reviewed the canine liver transplantation model as I/R injury model to delineate the intermediate filaments of the cytoskeleton that are probably the determinants in changing the phenotype of hepatocytes to cholangiocytes. Remarkably, this interesting feature seems to occur earlier than frank cholestasis. We speculate that I/R liver injury through a phenotypical switch of the hepatocytes may contribute to the poor outcome of the liver graft.

\section{Introduction}

Liver transplantation is an established therapy for both acute and chronic liver failure. However, graft dysfunction remains a problem affecting up to one-third of the recipients, despite reports of good to excellent long-term outcome. The organ dysfunction is considered multifactorial, but ischemia and reperfusion (I/R) injury is probably the most important contributing factor, although the detailed steps of pathogenesis are controversially debated. I/R injury is a major cause of liver graft dysfunction resulting in adenosine triphosphate (ATP) decrease, evidence of intracellular acidosis, and cell swelling of the hepatocytes. This aspect is also accompanied by or linked to bile duct loss or ductopenia, cholestasis, and biliary ductular proliferations in the posttransplant liver biopsy. However, biliary marker levels increase usually only 5-7 days after transplantation stimulating several fields of research in the last couple of decades. Cholestasis is associated with high morbidity and mortality in patients undergoing liver transplantation, and the steps reaching this state are not completely understood. We reviewed the canine liver transplantation model as I/R injury model to delineate in detail the intermediate filaments of the cytoskeleton that are probably the determinants in changing the phenotype of hepatocytes to cholangiocytes, which seems to be a posttransplant event occurring in the liver at an earlier stage than frank cholestasis. Here, we speculate that I/R liver injury through a phenotypical switch of the liver cells may contribute to the poor outcome of the liver graft.

\section{Liver Transplantation}

Liver transplantation is widely known as the most effective therapy for both acute and chronic liver failure [1]. In 1963, Thomas Starzl, an American surgeon, Northwestern University Medical School graduate with degrees in anatomy, neurophysiology, and medicine, was the first to perform the 
liver transplantation on a child suffering from biliary atresia [2]. Despite the significant success of liver transplantation, infection, poor graft function, and rejection are major problems that may still contribute to death of the transplanted patient or to deterioration of the graft. Reperfusion following long ischemia of liver during liver transplantation causes severe injury, which is now universally indicated as I/R injury [1]. I/R injury of liver is a major cause of morbidity and mortality in patients undergoing liver transplantation [3]. Several mechanisms and distinct pathways may involve I/R leading to both initial poor function and primary nonfunction of the liver allograft [4]. Three cytoskeletal proteins form a network that provide the cellular structure and fundamental integrity of the eukaryotic cells: microfilaments, microtubules, and intermediate filaments. The intermediate filament proteins have an important role in liver protection against mechanical and nonmechanical injury, which have been demonstrated in animal models. Overexpression of proteins and mutations of the corresponding keratin genes have been reported to contribute to several human oncological and nononcological diseases [5].

\section{Cytoskeleton and Keratins Are Involved in the Development of the Intrahepatic Biliary System (K19 and K7)}

Eukaryotic cells have a unique cytoplasmic structure labeled as cytoskeleton, which consists of three distinct kinds of cytoskeletal filaments, including microfilaments, microtubules, and intermediate filaments. Besides giving the rigidity of the cell and maintaining cell shape and borders or cell "scaffolding," cytoskeleton plays important and probably crucial roles in intracellular transport, cell division, gene regulation, and signal transduction of the genetic information $[6,7]$. Actin, which is widely accepted to be a highly conserved structure among different species, is the major protein that constitutes the microfilaments. There are three known classes of human actin gene that have been identified as $\beta$-, $\alpha$-, and $\gamma$-actin. Cell-cell interaction, signal transduction, cell shape maintenance, and cell motility are the central functions of actin [7]. Microtubules, which have a diameter of around $23 \mathrm{~nm}$, are protofilaments that are important for the intracellular transport, movement of cilia and flagella, mitotic spindle, and cell wall synthesis. Tubulins are the major component of microtubules. Both microfilaments and microtubules have several groups of binding proteins that modulate their stability and biological functions, such as signal transduction [7]. Intermediate filaments with around $10 \mathrm{~nm}$ of diameter are more stable than actin filaments and function in the maintenance of cell shape by carrying tension. Intermediate filaments, which include, for example, vimentin, glial fibrillary acidic protein, neurofilament proteins, keratins, and nuclear lamins, organize the internal 3D cell structure anchoring cyto-organelles and serving as structural scaffolds of the nucleus and cytoplasm. Cytokeratins (Ks) are the main protein family that constitutes the intermediate filaments with more than fifty members that have been identified [8]. Polypeptides of the
Ks are variants and have been divided into type I and type II, or acidic and basic, respectively [9]. Studies have shown that each epithelial cell has a distinct content of cytokeratins (i.e., expression of keratins is described as tissue-specific manner) playing a major role in the tissue identification of metastasis of unknown primary carcinomas [10]. Ks usually present in cells as heteropolymers pairs composed of type I (K9K20) and type II (K1-K8). Similar to microfilaments and microtubules, keratins have numbers of their preferential binding proteins. K8 (MW 52KD) and K18 (MW 45KD) are considered as the only Ks that are normally expressed in human hepatocytes [10]. Moreover, both K8 and K18 have been found to be expressed in early development stage of mouse embryos [11]. Immunohistochemical studies have revealed that cholangiocytes (bile duct cells) express K7 (54 $\mathrm{KD})$ and $\mathrm{K} 19(40 \mathrm{KD})$ in addition to K8 and K18 [12], and those Ks have been used as valid markers for both studies on development of the intrahepatic biliary system and for assessment of bile duct damage [9]. It has also been well established that cholangiocytes are derived from hepatoblasts found around portal vein [13]. These cells show a particularly intense expression of K8 and K18. As indicated above, K7 and K19 have been linked to development of the intrahepatic bile duct system in both humans and animal models. The expression of K19 is useful to identify primitive biliary cells, while the expression of $\mathrm{K} 7$ appears after 20 weeks of gestation in humans [13]. Both K7 and K19 are consistently expressed in the development of the intrahepatic bile duct with elevated levels until one month of postnatal age. Thus, in order to phenotypically switch hepatoblasts into cholangiocytes, these cells should firstly express K19 followed by expression of K7 [13]. However, it has been shown that hepatocytes might express $\mathrm{K} 7$ in response to different conditions, such as ductopenia and cholestasis [14], and, interestingly, it has been found that hepatocellular carcinoma cells expressing K19 are significantly associated with reoccurrence of neoplasm after transplantation [15]. A recent study has demonstrated that hepatocytes are frequently expressing K7 in case of chronic liver allograft rejection [14]. Sergi et al. have demonstrated that hepatocytes intensively expressed K7 and K19 early following cold ischemia in a canine isolated perfused liver transplantation model [16]. Furthermore, they demonstrated that bile duct cytokeratins are very useful markers to diagnose an early sign of cholestasis. It has become clear that cholestasis is significantly associated with high morbidity and mortality in patients undergoing liver transplantation. Therefore, investigations that lead to discover the mechanisms of the cholestasis are dramatically required.

\section{Ischemia/Reperfusion (I/R) Injury}

$\mathrm{I} / \mathrm{R}$ injury is a phenomenon that occurs when blood flow and oxygen delivery return to reperfused tissue [17]. It is considered one of the major causes of morbidity and mortality among patients undergoing liver transplantation [3]. I/R injury has been demonstrated in several diseases such as cerebrovascular diseases, peripheral vascular diseases, sepsis, myocardial infarction, and organ transplantation 
[18]. It involves several mechanisms, which lead to organ failure, circulatory dysfunction, and, finally, death of the transplanted patient [1]. Cellular mechanisms of I/R injury include a few essential cell cascades that suggest the role of activation of endothelial cells, Kupffer cells, reactive oxygen species (ROS), and polymorphonuclear leukocytes (PMN) or neutrophils in the pathogenesis of I/R injury [19]. There may also be mechanisms involving $\mathrm{T}$ lymphocytes that seem to have a key role in short- and long-term damage during $\mathrm{I} / \mathrm{R}$ injury. T lymphocytes act as mediator in the subacute inflammatory phase of neutrophilic recruitment following I/R injury [20]. Another important constituent of tissue injury following $\mathrm{I} / \mathrm{R}$ injury that needs to be emphasized is the production of oxygen free radicals (OFRs). These OFRs can arise from different sources, such as Kupffer cells, PMN [1], and xanthine oxidase (XO) that is the most significant source [21]. Several etiological factors are involved in I/R injury, including ATP reduction [16], activation of proteases, and alteration in the intracellular concentration of cytokines and chemokines [22], and cell swelling [16]. Investigations of posttransplantation surgery have indicated that reperfusion plays an essential role in primary graft nonfunction, which is one of the most serious complications of liver transplantation [23]. The incidence of primary graft dysfunction is up to $20 \%$ [16]. I/R injury also causes early organ failure up to $10 \%$ as well as increases the acute and chronic rejection [1]. Several studies demonstrated that during I/R injury, morphological changes of liver tissues occur, and these changes can have a prognostic significance.

\section{Intermediate Filaments of Cytoskeleton}

The intermediate filament (IF) cytoskeletal protein is one of the three major cytoskeletal proteins whose result is important in maintaining both cellular structure and integrity of eukaryotic cells. The other two filament proteins are microfilaments (MF) and microtubules (MT) [5]. Beside their cellular functions like cell motility, division, and stress responses, they also have an essential role in human diseases due to mutations in filament proteins. For example, mutation in actin microfilaments will cause cardiac and noncardiac myopathies [5]. IF proteins consist of five types of keratins according to structure of genome and composition of amino acid, IF proteins types I-IV are cytoplasmic, and type V IF contain nuclear lamins [7]. The keratins are obligating noncovalently heteropolymers because they consist of one of each type I and type II keratins as pairs [9]. Type I or "acidic" and type II or "neutral to basic" keratins are the largest group of IF proteins [7] classified as type I (K9-K20) and type II (K1-K8) [24]. Another type of IF proteins is type III IF that includes vimentin, peripherin, glial fibrillary acidic protein, and desmin of mesenchymal cells, peripheral neurons, glia cells and astrocytes, and muscle cells, respectively [7]. Type IV IF proteins include neurofilament proteins (NF-L, NF-M, and NF-H), synemin, nestin, syncoilin, and $\alpha$-internexin; lamins $\mathrm{A}-\mathrm{C}$ for type V IF proteins [24]. They are the major IF proteins in the liver [5]. As indicated above, the adult hepatocytes express K8 and K18 only compared to other epithelial cells that express 2 or more type I or type II keratins [9] such as bile duct, which expresses additional keratins K7 and K19 [8]. Furthermore, alteration of the cytoskeletal proteins leads to several diseases and disorders. The first disease to be discovered related to keratin mutation was epidermolysis bullosa simplex (EBS) with mutations in K5 and K14. White sponge nevus syndrome is another disease caused by mutations in K4 and K13. It affects noncornifying stratified squamous epithelia in mouth, esophagus, and anogenital mucosa [25]. Additionally, mutations in $\mathrm{K} 8$ and/or K18 lead to acute or chronic liver diseases [26]. Normally in hepatocytes, the microfilaments are distributed in plasma membrane and region of the bile canaliculus. Further studies indicated that reperfusion alone causes alteration of theses microfilaments (F-actin) leading to contraction of canaliculi, relocalization of enzymes and transporters of canaliculi, and increasing permeability of cellular tight junction [16]. Cholestatic liver disease occurs when there is a decrease in bile flow, which is an abnormal physiologic state, and retention of toxic bile acids [27]. All three filament cytoskeletal proteins are remarkably altered in cholestatic liver disease. Clear cytoplasmic hepatocytes (cholate stasis) presented in cirrhotic nodules and showed a decrease in keratin IF network in the cytoplasm [28]. Studies on mice reported that keratin was overexpressed in epithelia of bile duct due to ligation of bile duct and in hepatocytes. In cholestatic liver disease, bile duct epithelial-type keratin (K7 and K19) was expressed in hepatocytes, too. Intermediate hepatocytes express $\mathrm{K} 7$, while reactive bile ductules express K7 and K19 [28]. In post-transplant period, morphological changes in liver tissue have also been demonstrated. Keratins of biliary type were detected in the hepatocytes in the early period of I/R injury. Moreover, bile canaliculus progressively dilate after ischemia, and a change of the microvillous integrity is demonstrable [16].

\section{Canine Liver Transplantation Model}

The liver is one of the organs with an incredible capacity for in vivo tissue engineering which allow restoration of the liver architecture and reestablishment of certain vital functions. The study of liver regeneration in humans arise ethical issues, and it is difficult to carry out because of a plethora of heterogeneous liver lesions. Accordingly, using experimental animal models is more useful and helpful for studying liver regeneration [29]. In vitro studies need to be followed by in vivo ones to simulate the interaction between liver cell populations. In our opinion, the use of large animals such as dog, pig, or sheep is more advantageous than small animals like rat or mouse; large animals are similar to human beings in their physiology and anatomy, and techniques of microsurgery are not necessary to carry out determinate experiments [29]. Only few studies have been carried out on $\mathrm{I} / \mathrm{R}$ animal models in liver [21]. The canine liver model following $\mathrm{I} / \mathrm{R}$ injury has been used as a liver transplantation model to study excretion of bile and intrahepatic intermediate filaments expression involved in morphological changes of the biliary system [16]. To the best of our knowledge, in a canine isolated and 
perfused liver model, there was the first clear-cut evidence of cholestatic changes starting early following cold ischemia despite prompt recovery of the bile flow. According to some other authors, the use of the canine liver transplantation model seems to allow a better vascular perfusion under ex vivo conditions in contrast to the rat model [5]. Sergi et al.'s study revealed an ischemia-dependent impairment of an experimental biliary dye excretion during early stage of reperfusion and a progressive cytokeratin expression of biliary type in the hepatocytes despite a prompt recovery of the bile flow after I/R. The recovery of the bile output after I/R represents a key event in LT. The nonstimulated bile flow rate was unaffected by cold ischemia up to $10 \mathrm{~h}$ in our canine isolated perfused liver model, but the decrease of the biliary dye excretion during early stage of reperfusion correlated with the peak output rate of the dye across the canalicular membrane. The transhepatic transit time was prolonged with increasing ischemia time. Remarkably, the dye uptake at the sinusoidal membrane was not affected by cold ischemia, leading to a possible accumulation of toxic compounds in the hepatocytes. Hepatic elimination of the dye following $\mathrm{I} / \mathrm{R}$ has been analyzed mainly by plasma clearance [4]. Very importantly was the study of the hepatic elimination of the dye after a bolus injection by compartmental spectrophotometric analysis both in the perfusate by transhepatic sampling and in the bile. In fact, this reflects the "effective" vectorial hepatocellular transport. Differential mechanisms can be suggested for the impaired biliary excretion of organic anions following I/R. Previously, a positive correlation between biliary dye excretion, viability of the graft, and hepatic ATP content has been found in cholestatic liver disease, and reduced biliary dye excretion has been attributed to the lack of ATP [17]. ATP content does not seem to be the main limiting factor for the hepatocellular transport following I/R. An impairment of the intracellular transport or a decreased transport rate across the canalicular membrane might play a major role as contributing factor. The step across the canalicular membrane generally represents the rate-limiting one in the hepatocellular transport [18]. In the canine liver transplantation model, the flow in the portal vein was continuous, because roller pump, oxygenator, and heat exchanger in the perfusion line were connected in series with the roller pump upstream. The oxygen content of the saline reperfusion solution was $2 \%$ in volume ( $10 \%$ of arterial hepatic blood and about $15 \%$ of the portal venous blood). The $\mathrm{pO}_{2}$ values were almost five times higher than in blood. Thus, one can expect to have reactiveoxygen-species- (ROS-) linked damage at the beginning of the reperfusion after 8 or $10 \mathrm{~h}$ of ischemia. However, the excellent reperfusion results of the control group and data of the $2 \mathrm{~h}$ ischemia group indicate that this is not a general problem, but may be an ischemia-time-linked problem. High $\mathrm{O}_{2}$ partial pressure in the liver stimulates ROS formation after extensive long ischemia times, and this may be one of the most important limiting factors of the ischemia tolerance [22]. Electron microscopy studies in hepatocytes revealed microfilaments, which are distributed along the plasma membrane and in the region of the bile canaliculus. As indicated above, cytoskeleton filaments are also essential for the maintenance of cell shape, bile canalicular architecture, and integrity of the cellular tight junctions. The microfilaments network surrounding the canalicular pole and extending into the canalicular microvilli is damaged during cholestasis. Reperfusion, but not ischemia, has been considered faulty, inducing an alteration of F-actin microfilaments, suggesting an impairment of the canalicular contraction, an increase of the tight junction permeability, and a probable relocalization of canalicular enzymes and transporters. Bile canalicular size change as detected in our study may be coincident with the reorganization of the pericanalicular filaments and the colocalization of actin and myosin. In fact, contraction in this study has been associated with shortening and/or twisting of bile canaliculi [5].

\section{Summary}

Liver transplantation is an established therapy for both acute and chronic liver failure. However, graft dysfunction remains a problem affecting up to one-third of the recipients, despite good to excellent long-term outcome. The organ dysfunction is considered multifactorial, but I/R injury is probably the most important contributing factor. The detailed steps of the pathogenesis of $I / R$ injury continue to be a topic of vivid debate. I/R injury is a major cause of liver graft dysfunction resulting in ATP decrease, intracellular acidosis, and cell swelling of the hepatocytes. This feature is also linked to bile duct loss or ductopenia, cholestasis, and biliary ductular proliferations in the liver biopsy. However, the plasmatic levels of biliary markers increase usually only 57 days after transplantation prompting on-going research to address new markers of early liver damage. This could also be a stimulus to support more grant approvals for liver posttransplantation studies. Cholestasis is associated with high morbidity and mortality in patients undergoing liver transplantation, but the steps reaching this state are still deemed elusive. We reviewed the canine LT model as an I/R injury model and reviewed the intermediate filaments of the cytoskeleton that are probably the determinants in changing the phenotype of hepatocytes to cholangiocytes. In fact, this phenotypic switch of the liver cells, which is also sometimes called pseudo- or neoacinar transformation in diseases with cholestasis [30], seems to occur at a stage earlier than frank cholestasis. We emphasized that I/R injury through this peculiar phenotypic switch of the hepatocytes may be a milestone to investigate the pathways contributing to the poor outcome of the liver graft. Reperfusion injury following ischemia affects graft function in liver transplantation by induction of phenotypical changes in hepatocellular keratins. Keratin phenotype of hepatocytes leads to hepatocellular damage that is intimately connected to several intracellular processes, which are under intense investigation in our laboratory, and the appropriate choice of an animal model is crucial in forwarding research in very complex areas requiring not only human, but also comparative (veterinary) pathology knowledge. 


\section{Acknowledgments}

The authors are very grateful to the Saudi Cultural Bureau, Ottawa, ON, Canada, for the financial support of two graduate students (R. Abdualmjid and Y. Abuuetabh.).They are also very grateful to Tyrolean Cancer Research Fund, Innsbruck, Austria and University of Alberta for some extraand intramural funding, respectively. This work is dedicated to the memory of Professor Dr. h.c. Herwart F. Otto, formerly chairman and emeritus professor of pathology at the Institute of Pathology, Ruperto-Carola University of Heidelberg, Germany.

\section{References}

[1] J. W. Kupiec-Weglinski and R. W. Busuttil, "Ischemia and reperfusion injury in liver transplantation," Transplantation Proceedings, vol. 37, no. 4, pp. 1653-1656, 2005.

[2] T. E. Starzl, T. L. Marchioro, K. N. Vonkaulla, G. Hermann, R. S. Brittain, and W. R. Waddell, "Homotransplantation of the liver in humans," Surgery, Gynecology \& Obstetrics, vol. 117, pp. 659-676, 1963.

[3] F. Dünschede, K. Erbes, A. Kircher et al., "Reduction of ischemia reperfusion injury after liver resection and hepatic inflow occlusion by $\alpha$-lipoic acid in humans," World Journal of Gastroenterology, vol. 12, no. 42, pp. 6812-6817, 2006.

[4] P. A. Clavien, P. R. C. Harvey, and S. M. Strasberg, "Preservation and reperfusion injuries in liver allografts: an overview and synthesis of current studies," Transplantation, vol. 53, no. 5, pp. 957-978, 1992.

[5] M. B. Omary, N. O. Ku, and D. M. Toivola, "Keratins: guardians of the liver," Hepatology, vol. 35, no. 2, pp. 251-257, 2002.

[6] E. Frixione, "Recurring views on the structure and function of the cytoskeleton: a 300-year epic," Cell Motility and the Cytoskeleton, vol. 46, no. 2, pp. 73-94, 2000.

[7] N. O. Ku, X. Zhou, D. M. Toivola, and M. B. Omary, "The cytoskeleton of digestive epithelia in health and disease," American Journal of Physiology, vol. 277, no. 6, pp. G1108-G1137, 1999.

[8] P. Strnad, C. Stumptner, K. Zatloukal, and H. Denk, "Intermediate filament cytoskeleton of the liver in health and disease," Histochemistry and Cell Biology, vol. 129, no. 6, pp. 735-749, 2008.

[9] P. Van Eyken and V. J. Desmet, "Cytokeratins and the liver," Liver, vol. 13, no. 3, pp. 113-122, 1993.

[10] R. Moll, W. W. Franke, and D. L. Schiller, "The catalog of human cytokeratins: patterns of expression in normal epithelia, tumors and cultured cells," Cell, vol. 31, no. 1, pp. 11-24, 1982.

[11] B. W. Jackson, C. Grund, and E. Schmid, "Formation of cytoskeletal elements during mouse embryogenesis. Intermediate filaments of the cytokeratin type and desmosomes in preimplantation embryos," Differentiation, vol. 17, no. 3, pp. 161-179, 1980.

[12] P. Van Eyken, R. Sciot, B. Van Damme, C. De Wolf-Peeters, and V. J. Desmet, "Keratin immunohistochemistry in normal human liver. Cytokeratin pattern of hepatocytes, bile ducts and acinar gradient," Virchows Archiv, vol. 412, no. 1, pp. 6372, 1987.

[13] P. Van Eyken, R. Sciot, F. Callea, K. Van der Steen, P. Moerman, and V. J. Desmet, "The development of the intrahepatic bile ducts in man: a keratin-immunohistochemical study," Нераtology, vol. 8, no. 6, pp. 1586-1595, 1988.
[14] A. M. Bellizzi, R. D. LeGallo, J. C. Boyd, and J. C. Iezzoni, "Hepatocyte cytokeratin 7 expression in chronic allograft rejection," American Journal of Clinical Pathology, vol. 135, no. 2, pp. 238-244, 2011.

[15] A. Durnez, C. Verslype, F. Nevens et al., "The clinicopathological and prognostic relevance of cytokeratin 7 and 19 expression in hepatocellular carcinoma. A possible progenitor cell origin," Histopathology, vol. 49, no. 2, pp. 138-151, 2006.

[16] C. Sergi, W. Gross, M. Mory, M. Schaefer, and M. M. Gebhard, "Biliary-type cytokeratin pattern in a canine isolated perfused liver transplantation model," Journal of Surgical Research, vol. 146, no. 2, pp. 164-171, 2008.

[17] E. E. Montalvo-Jave, T. Escalante-Tattersfield, J. A. OrtegaSalgado, E. Piña, and D. A. Geller, "Factors in the Pathophysiology of the Liver Ischemia-Reperfusion Injury," Journal of Surgical Research, vol. 147, no. 1, pp. 153-159, 2008.

[18] G. Bilbao, J. L. Contreras, D. E. Eckhoff et al., "Reduction of ischemia-reperfusion injury of the liver by in vivo adenovirusmediated gene transfer of the antiapoptotic Bcl-2 gene," Annals of Surgery, vol. 230, no. 2, pp. 185-193, 1999.

[19] C. Fondevila, R. W. Busuttil, and J. W. Kupiec-Weglinski, "Hepatic ischemia/reperfusion injury-a fresh look," Experimental and Molecular Pathology, vol. 74, no. 2, pp. 86-93, 2003.

[20] R. M. Zwacka, Y. Zhang, J. Halldorson, H. Schlossberg, L. Dudus, and J. F. Engelhardt, "CD4+ T-lymphocytes mediate ischemia/reperfusion-induced inflammatory responses in mouse liver," Journal of Clinical Investigation, vol. 100, no. 2, pp. 279-289, 1997.

[21] H. Ajamieh, N. Merino, E. Candelario-Jalil et al., "Similar protective effect of ischaemic and ozone oxidative preconditionings in liver ischaemia/reperfusion injury," Pharmacological Research, vol. 45, no. 4, pp. 333-339, 2002.

[22] V. Kohli, W. Gao, C. A. Camargo, and P. A. Clavien, "Calpain is a mediator of preservation-reperfusion injury in rat liver transplantation," Proceedings of the National Academy of Sciences of the United States of America, vol. 94, no. 17, pp. 9354-9359, 1997.

[23] L. Benkoël, F. Dodero, J. Hardwigsen et al., "Effect of ischemiareperfusion on bile canalicular F-actin microfilaments in hepatocytes of human liver allograft: image analysis by confocal laser scanning microscopy," Digestive Diseases and Sciences, vol. 46, no. 8, pp. 1663-1667, 2001.

[24] M. Hesse, T. M. Magin, and K. Weber, "Genes for intermediate filament proteins and the draft sequence of the human genome: novel keratin genes and a suprisingly high number of pseudogenes related to keratin genes 8 and 18," Journal of Cell Science, vol. 114, no. 14, pp. 2569-2575, 2001.

[25] A. D. Irvine and W. H. I. McLean, "Human keratin diseases: the increasing spectrum of disease and subtlety of the phenotype-genotype correlation," British Journal of Dermatology, vol. 140, no. 5, pp. 815-828, 1999.

[26] N. O. Ku, R. Gish, T. L. Wright, and M. B. Omary, "Keratin 8 mutations in patients with cryptogenic liver disease," New England Journal of Medicine, vol. 344, no. 21, pp. 1580-1587, 2001.

[27] G. F. Hutchins and J. L. Gollan, "Recent developments in the pathophysiology of cholestasis," Clinics in Liver Disease, vol. 8, no. 1, pp. 1-26, 2004.

[28] K. Zatloukal, C. Stumptner, A. Fuchsbichler et al., "The keratin cytoskeleton in liver diseases," Journal of Pathology, vol. 204, no. 4, pp. 367-376, 2004.

[29] D. Palmes and H. U. Spiegel, "Animal models of liver regeneration," Biomaterials, vol. 25, no. 9, pp. 1601-1611, 2004. 
[30] L. Dorn, L. F. Menezes, G. Mikuz, H. F. Otto, L. F. Onuchic, and C. Sergi, "Immunohistochemical detection of polyductin and co-localization with liver progenitor cell markers during normal and abnormal development of the intrahepatic biliary system and in adult hepatobiliary carcinomas," Journal of Cellular and Molecular Medicine, vol. 13, no. 7, pp. 1279-1290, 2009. 


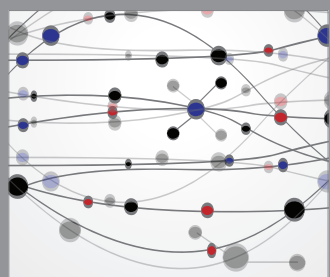

The Scientific World Journal
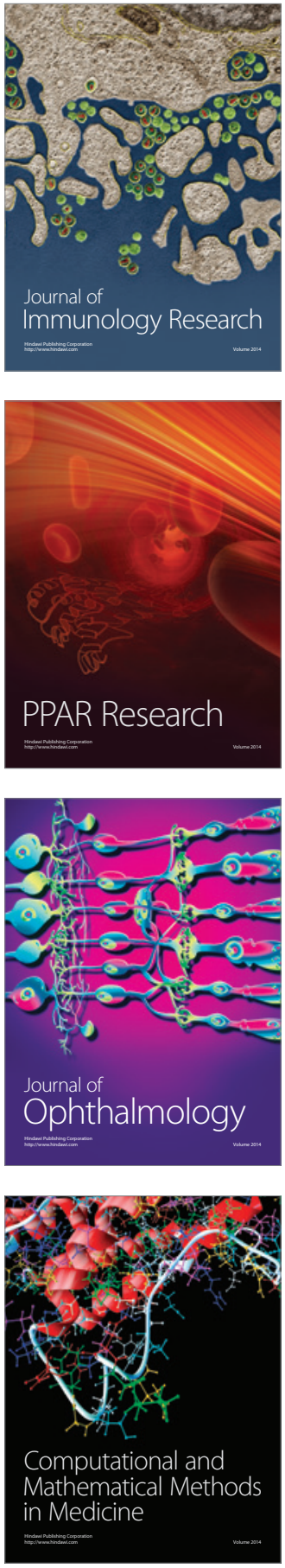

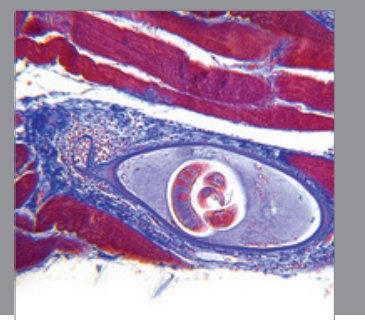

Gastroenterology

Research and Practice
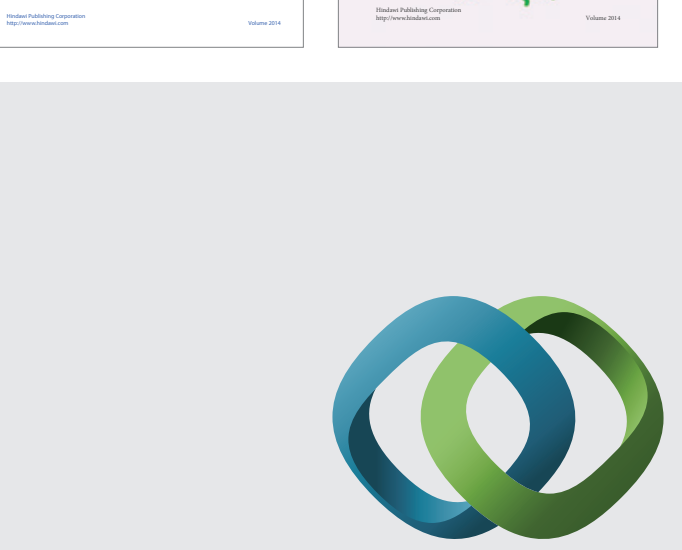

\section{Hindawi}

Submit your manuscripts at

http://www.hindawi.com
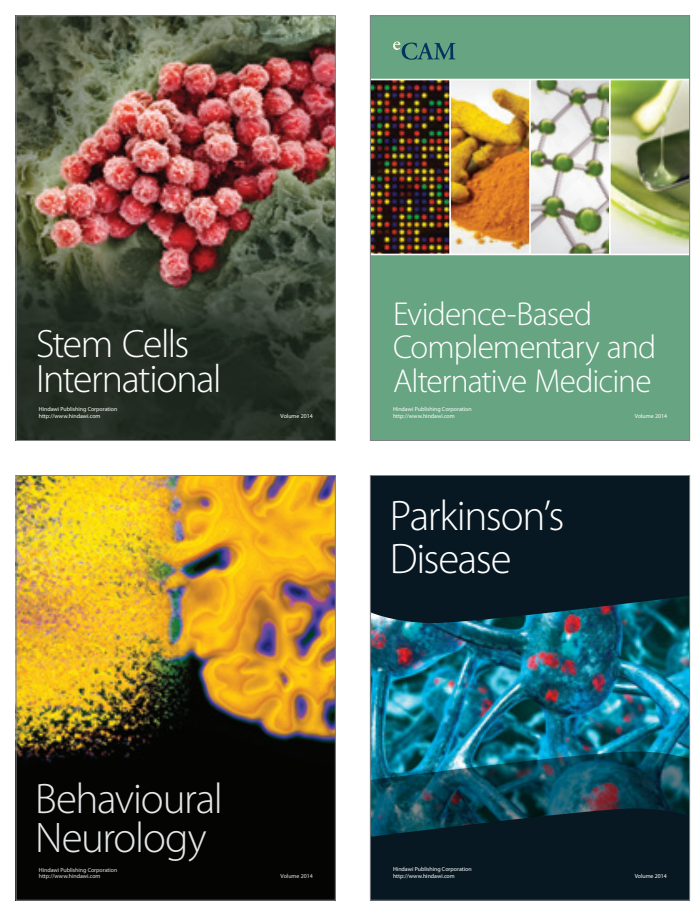

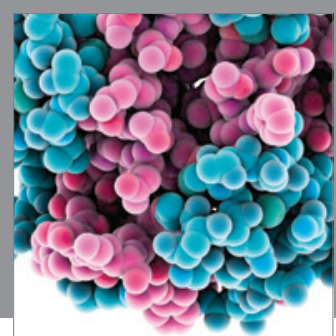

Journal of
Diabetes Research

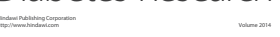

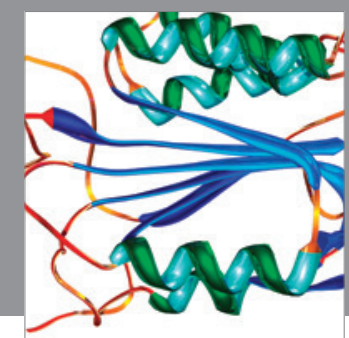

Disease Markers
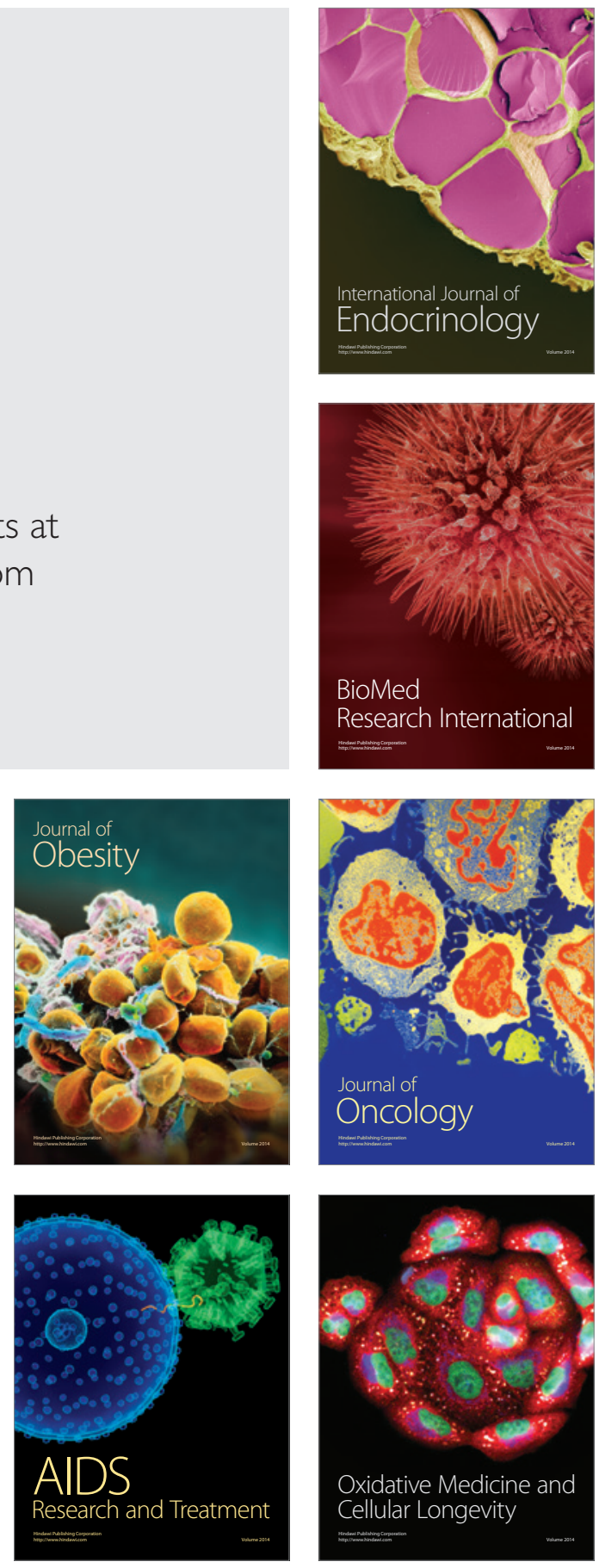